\title{
Two Rare Syndromic Syndactyly Cases in Neonates
}

\author{
Pooja Prabhakar Kamath ${ }^{1}$ \\ ${ }^{1}$ Department of Pediatrics, V. N. Desai Hospital, Mumbai, \\ Maharashtra, India
}

Int J Recent Surg Med Sci 2021;7:91-93.

\begin{abstract}
Address for correspondence Pooja Prabhakar Kamath, MBBS, MD, Ultra Co Op Hsg Soc, Lt Dilip Gupte Marg, Mahim, Mumbai 400016, Maharashtra, India (e-mail: drpoojakamath@gmail.com).
\end{abstract}

\begin{abstract}
Keywords

- syndactyly

- otopalatodigital

syndrome

- orofaciodigital

syndrome

Introduction Isolated findings of syndactyly are benign. However, syndactyly can be associated with rare syndromes that need to be diagnosed for further management and for genetic counseling.

Methods We present two cases of syndromic syndactyly in neonates. The first case is a 13-day-old female neonate with dysmorphic features. The neonate had clinical features of prominent forehead, hypertelorism, widely separated sagittal and metopic sutures, down-slanting eyes, low set ears, depressed nasal bridge, micrognathia, cleft palate, pectus excavatum, brachydactyly, and syndactyly of the second to fourth fingers bilaterally in upper limbs and in lower limbs.

The second case is a 10-day-old male neonate with dysmorphism in the form of cleft alveolar ridge and palate, hyperplastic frenula, hypoplastic alar cartilage, syndactyly of the left hand, clinodactyly of the left lower limb toes, and amniotic bands.

Discussion Case 1 was diagnosed as otopalatodigital syndrome because of the characteristic clinical features. This is a rare syndrome associated with syndactyly that often goes undiagnosed. Otopalatodigital syndrome spectrum disorders comprise of four phenotypically related conditions: otopalatodigital syndrome types 1 and 2, frontometaphyseal dysplasia, and Melnick-Needles syndrome. As it is associated with $\mathrm{x}$-linked inheritance, its severity is more in males.

Case 2 was diagnosed to have orofaciodigital syndrome because of the characteristic clinical features. It is another rare syndrome associated with syndactyly having abnormalities in the development of the oral cavity, face, and digits along with intellectual disability and renal system impairment.

Conclusion There are fewer publications on these syndromes as they are rare and diagnosis is difficult. Recognizing these syndromes is key to further management and for genetic counseling.
\end{abstract}

\section{Introduction}

Syndactyly is the most common congenital abnormality of the hand, occurring 1 per 2,000 to 3,000 births. Isolated cases of syndactyly are benign, familial, or hereditary, and generally do not require any treatment. However, those associated with syndromes have to be investigated for appropriate management. Here we present two syndromic cases of syndactyly.

published online May 29, 2021
DOI https://doi.org/

10.1055/s-0041-1730256

ISSN 2455-7420

\section{Case Summary}

\section{Case 1}

The first case was a 13-day-old female neonate with dysmorphic features, born to P2L2 mother with nonconsanguinous marriage and nonsyndromic parents. On examination, the neonate was found to have prominent forehead, hypertelorism, widely separated sagittal and metopic sutures,

\section{(C) 2021. Medical and Surgical Update Society.}

This is an open access article published by Thieme under the terms of the Creative Commons Attribution-NonDerivative-NonCommercial-License, permitting copying and reproduction so long as the original work is given appropriate credit. Contents may not be used for commercial purposes, or adapted, remixed, transformed or built upon. (https://creativecommons.org/licenses/by-nc-nd/4.0/).

Thieme Medical and Scientific Publishers Pvt. Ltd. A-12, 2nd Floor, Sector 2, Noida-201301 UP, India 
down-slanting eyes, low set ears, depressed nasal bridge, micrognathia, cleft palate, pectus excavatum, brachydactyly, and syndactyly of the second to fourth fingers ( - Fig. 1) bilaterally in the upper and lower limbs. The neonate was diagnosed as otopalatodigital syndrome. Ultrasonography of the skull was done to rule out the presence of hydrocephalus or other malformations, which was normal. Abdominal ultrasonography, 2D echocardiogram, and brainstem evoked response audiometry (BERA) were also normal. The second case was an full term normal delivery, $2.7-\mathrm{kg}$ neonate referred to us on day 10 of life with dysmorphism in the form of cleft alveolar ridge and palate, hyperplastic frenula, hypoplastic alar cartilage, syndactyly of the left hand, clinodactyly of the left toes, and amniotic bands. On auscultation, a systolic murmur was present following which 2D echocardiogram was done, which revealed an atrial septal defect, ventricular septal defect with severe pulmonary hypertension, and tricuspid valve dysplasia. Magnetic resonance imaging (MRI) of the brain was within normal limits. So our neonate had features of orofaciodigital syndrome (OFDS).

Both the patients were born of nonconsanguinous marriage to P2L2 mothers and had nonsyndromic parents. MRI of the brain for the first case and sequence analysis for both are planned on follow-up.

\section{Discussion}

Syndactyly is one of the most frequent hereditary limb malformations. ${ }^{1}$ Syndactyly, or webbing of the fingers, can be complete or partial; it can be simple, with only skin binding the fingers together, or complicated, when there are fused bones, extra bones, or shared structures, such as nerves, vessels, or nails.

Syndactyly has varied clinical malformation. It can exist in syndromic or nonsyndromic forms. The presentations may vary from unilateral or bilateral and symmetrical or asymmetrical to complete or incomplete and cutaneous or bony. ${ }^{2}$

It occurs due to failure of differentiation into individual appendages occurring during the sixth and eighth weeks of

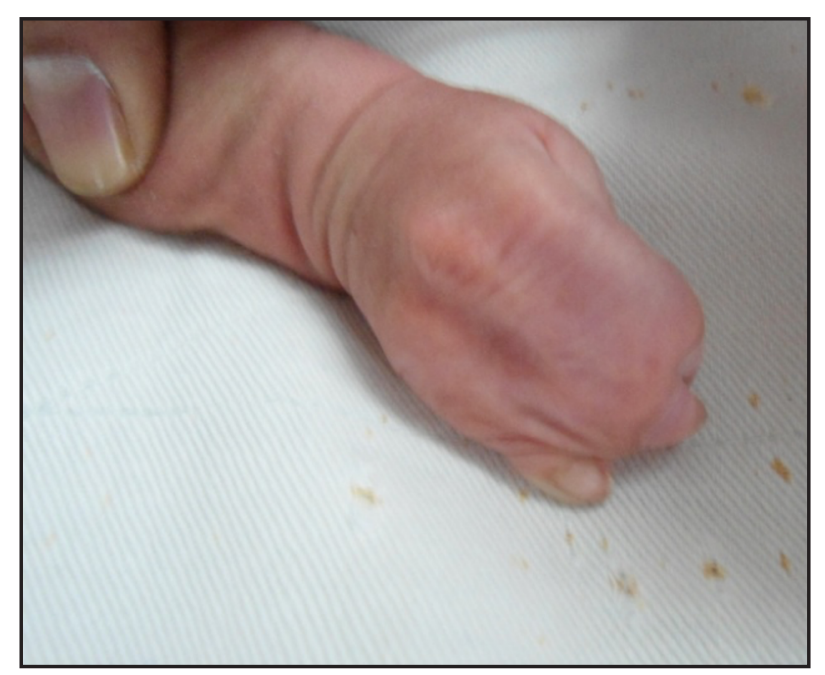

Fig. 1 Syndactyly of the right hand. embryologic development with a male-to-female ratio of 2:1. In all, 10 to $40 \%$ of cases are familial with variable penetrance, sporadic, or as part of common syndromes like Apert's syndrome, Poland's syndrome, Holt-Oram syndrome, OFDS, and otopalatodigital syndrome.

In case 1 , the patient had, along with syndactyly, prominent forehead, hypertelorism, widely separated sagittal and metopic sutures, down-slanting eyes, low set ears, depressed nasal bridge, micrognathia, cleft palate, pectus excavatum, and brachydactyly as a result of which our patient appeared to be a case of otopalatodigital spectrum disorder.

The baby was given an obturator to overcome the cleft palate to establish effective breastfeeding. The mother was taught to feed the baby in sitting position as babies are prone to aspiration. Occupational therapist reference was obtained for oromotor stimulation.

In later infancy, this baby will require plastic surgery for reconstruction of the cleft palate and digits. The mother was counseled about the requirement of speech therapy to overcome the speech deficits that occur with many patients with a cleft palate.

In case 2, the patient had, along with syndactyly, other features of dysmorphism in the form of cleft alveolar ridge and palate, hyperplastic frenula (-Fig. 2), hypoplastic alar cartilage, clinodactyly of the left toes, and amniotic bands. Thus, our second case appeared to be a case of OFDS.

The neonate was given an obturator for effective breastfeeding and the mother was taught to feed the baby in sitting position. After taking the opinion of a cardiologist, the patient was started on tab sildenafil and oxygen therapy to reduce pulmonary hypertension. In this baby, a further follow-up with 2D echocardiogram was suggested after 3 months, on the basis of which a cardiac catheterization study would be planned and then definitive surgical procedure would be decided upon. The baby was referred to plastic surgery for syndactyly, clinodactyly, and amniotic band correction.

\section{Otopalatodigital Syndrome}

This is a rare skeletal dysplasias characterized by anomalous ossification and skeletal defects (axial and appendicular) with a phenotypic spectrum comprising otopalatodigital syndrome types I and II, Melnick-Needles syndrome, and frontometaphyseal dysplasia. Its incidence is 1 in 100,000 . Types I (milder form) and II are rare X-linked genetic disorders with complete expression only in males and mild affection in females.

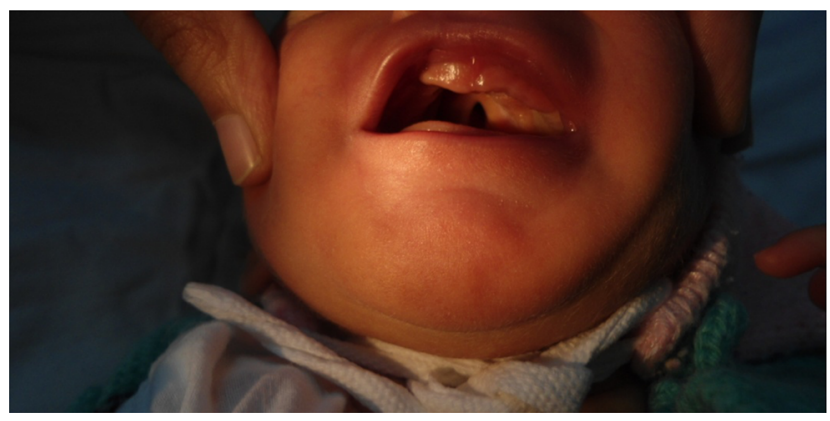

Fig. 2 Hyperplastic frenula and cleft palate. 


\section{Otopalatodigital Syndrome Type 1}

In otopalatodigital syndrome type 1 , most manifestations are present at birth; females can present with severity similar to affected males, although some have only mild manifestations.

Otopalatodigital syndrome type 1 has features such as hypertelorism, prominent brow ridges, large fontanelle, cleft palate, hearing impairment, hypodontia, and skeletal anomalies like syndactyly, thoracic hypoplasia, deformities of long bones, campomelia. ${ }^{3}$

Otopalatodigital syndrome type 1 is the mildest phenotype in males of all four otopalatodigital syndrome spectrum disorders.

Clinical manifestations in females are usually milder compared with males, but occasionally can be as severely affected as males.

\section{Otopalatodigital Syndrome Type 2}

The skeletal dysplasia in otopalatodigital syndrome type 2 is more severe than in otopalatodigital syndrome type 1. Additional extra-skeletal anomalies being brain malformations like hydrocephalus, heart defects like atrial septal defect and ventricular septal defect, abdominal defects like omphalocele, dysplastic kidney, intestinal malformation, obstructive uropathy, and malformations in the tracheobronchial tree. Most of the male babies do not survive beyond infancy. ${ }^{4}$ Male babies who live beyond the first year of life are usually developmentally delayed and require respiratory support and assistance with feeding. ${ }^{5}$

On further investigation in case 1, ultrasonography of the skull and abdomen, 2D echocardiograph, and BERA were found to be normal; therefore, our clinical diagnosis was otopalatodigital syndrome type 1 .

\section{Orofaciodigital Syndrome}

It consists of distinctive rare genetic disorders of heterogenous inheritance. The incidence is 1 in 50,000 to $250,000 .{ }^{6}$ There are abnormalities in the development of the oral cavity, face, and digits along with intellectual disability and renal system impairment. OFDS type I is the most common of all of these disorders. OFDS types 1 and 2 are comparatively studied in detail. OFDS type 1 comprises $75 \%$ of cases with X-linked dominant inheritance. Males with OFDS type 1 almost always die in utero, whereas females are born with variable phenotypic manifestations

OFDS, characterized by syndactyly, clinodactyly, cleft palate, lobulated tongue, hypertelorism, micrognathia, hypoplastic nose, intellectual disability, Dandy-Walker malformation, corpus callosal agenesis, polycystic kidney disorder, congenital heart disorders, thoracic deformities, and growth retardation. ${ }^{7}$

In case 2, 2D echocardiography, performed in view of systolic murmur, revealed an atrial septal defect/ventricular septal defect with severe pulmonary hypertension and tricuspid valve dysplasia. MRI of the brain was within normal limits.

Parents of both patients were counseled about the syndrome and the need for molecular genetic testing for confirmation of the disorder, its long-term prognosis, and its effect on future pregnancies. Such patients are in need of various surgical interventions as part of treatment and rehabilitation. A multidisciplinary approach is required for rehabilitation such as hearing aids, orthotics, speech therapy, occupational therapy, and palliative feeding procedures.

\section{Conclusion}

Diagnoses of both otopalatodigital and orofaciodigital disorders are extremely difficult because they are rare and there is lack of adequate research.

Genetic counseling and carrier testing are of great importance to prevent the occurrence of such syndromes in future pregnancies as both syndromes in severe forms are lethal in males. Syndactyly that is associated with dysmorphism should be thoroughly investigated.

Key to diagnosis is molecular genetic testing. There is no cure for these genetic disorders, but treatment of the associated anomalies like cardiac defects, renal defects, cleft palate, etc., should be undertaken as supportive management.

\section{Conflict of Interest}

None declared.

\section{References}

1 Tonkin MA. Failure of differentiation part I: syndactyly. Hand Clin 2009;25(2):171-193

2 Ahmed H, Akbari H, Emami A, Akbari MR. Genetic overview of syndactyly and polydactyly. Plast Reconstr Surg Glob Open 2017;5(11):e1549

3 Vasikarla M, Sharma DK, Murki S, Pratap T. Otopalatodigital syndrome type 1: a rare skeletal disorder. Sri Lanka J Child Health 2016;45(1):44-45

4 Robertson SP. Otopalatodigital syndrome spectrum disorders: otopalatodigital syndrome types 1 and 2, frontometaphyseal dysplasia and Melnick-Needles syndrome. Eur J Hum Genet 2007;15(1):3-9

5 Robertson S. X-linked otopalatodigital spectrum disorders; 2005 [updated 2019 Oct 3]. In: Adam MP, Ardinger $\mathrm{HH}$, Pagon RA, Wallace SE, Bean LJH, Stephens K, Amemiya A, eds. GeneReviews [Internet]. Seattle, WA: University of Washington, Seattle; 1993-2020

6 Gorlin RJ, Anderson VE, Scott CR. Hypertrophied frenuli, oligophrenia, famflial trembling and anomalies of the hand. Report of four casesin one family and a forme fruste in another. $\mathrm{N}$ Engl J Med 1961;264:486-489

7 Toriello HV, Franco B, Bruel AL, et al. Oral-facial-digital syndrome type I; 2002 Jul 24 [Updated 2016 Aug 4]. In: Adam MP, Ardinger HH, Pagon RA, et al., eds. GeneReviews [Internet]. Seattle, WA: University of Washington; 1993-2020. Available at: https://www.ncbi.nlm.nih.gov/books/NBK1188/ 